\title{
Research on Integrated Monitoring System of High-voltage Tower
}

\author{
Huichun Huang \\ Southeast University \\ Nanjing, China \\ huanghuichun@seu.edu.cn
}

\author{
Wei Jiang \\ Southeast University \\ Nanjing, China \\ jiangwei@seu.edu.cn
}

\author{
Renjie Hu \\ Southeast University \\ Nanjing, China \\ hurenjie@seu.edu.cn
}

\begin{abstract}
The monitoring and stable operation of high-voltage tower is an important direction of strong grid research. Transmission monitoring includes the forecast of early faults and real-time state estimation. There is no domestic report on related research. Some simulation in laboratory had been done and little been reported. The research review was carried out in accordance with existing knowledge and research progress summary, using wireless transmission and self-generating as example, to build high-voltage tower monitoring system including power management systems, sensor systems, data processing systems, signal transmission system and remote monitoring system.
\end{abstract}

Keywords-High-voltage Tower; MonitoringSystems; Sensor Systems

\section{INTRODUCTION}

High-voltage transmission lines had been used more and more with high speed development of economy. Because of the widely distributed and complex environment, monitoring and maintenance of the transmission lines is very difficult. The main factors affecting the safety of electrical equipment mainly from two aspects, the human factor like theft, climbing tower, illegal construction and natural factors like lightning, storms, snow and landslides.

The most important function of smart grid [1] is to achieve stable running of the grid by reducing the risk of large-scale blackouts, and improving self-healing ability. Grid self-healing capabilities include remote monitoring and analysis of equipment on the grid, and automatic evaluation of transmission and consumption system. Those ensure the safety of the power grid, and curb the occurrence of the blackout. System monitoring and stable operation of the high-voltage tower became an important direction of the strong grid research at home and abroad.

Currently the monitoring of high-voltage tower and transmission line uses the helicopter patrol line. The biggest flaw of this method is safety. The helicopters are affected by the weather, and cost much. Began in the late 1980s, inspection robot had been researched abroad. The robot system was complex, and the energy consumption was also a serious problem. In recent years, with the development of sensor networks, power electronics, power generation, energy storage and wireless communications [2] technology, the application of these techniques to solve the security problems of the high-voltage tower has become possible.

\section{ARCHITECTURE OF HIGH-VOLTAGE TOWER MONITORING SYSTEM}

Monitoring of high-voltage tower is a kind of to remote monitoring, including wired and wireless communication. Due to taking power in the transmission line is prohibited in the high voltage transmission system, the power supply mode of the high-voltage tower monitoring system can use the self-generating or dedicated power line. The following example, using wireless transmission and self-generating to construct the high voltage tower system, is shown in Figure 1. The subsystems of the monitoring system mainly include follows: power management system, sensor system, data processing system, signal transmission system and remote monitoring system.

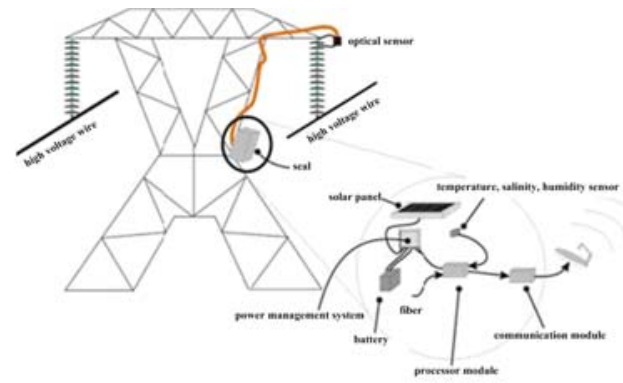

Figure 1. high-voltage tower monitoring system diagram

\section{HIGH-VOLTAGE TOWER MONITORING SYSTEM}

\section{A. Power system}

Mature ways of power supply include solar power, wind power and dedicate power. Monitoring of high-voltage tower has characteristics of ultra-long distance with linear distribution, and high-voltage tower monitoring system mainly use the DC supply. So solar and wind power generation is the preferred power supply.

\section{B. The sensor system}

The sensor system is a key component of the high-voltage tower monitoring system. The environmental parameters of high-voltage tower, and the mechanics parameters with aging of transmission line and tower require systematic detecting.

1) The mechanics parameters and aging of the tower body

Monitoring of the tower body comprises the monitoring of the internal stress of the metal structure, the degree of 
metal aging. Currently, electrical measuring method using resistance strain gauge stress-strain measurements is a common method for test, analysis and evaluation the reliability and safety of the engineering structural design, manufacturing and assembly. It is widely used in aviation, machinery, vehicle and civil engineering fields [3].

Commonly used methods of aging testing (NDT) are eddy current testing and ultrasonic testing.

\section{a) Eddy current sensor}

Eddy current testing is quickly and particularly suitable for tube, rod detection. It has high sensitivity for surface and near-surface defects. It can evaluate the defects of different sizes even under high temperature. Profile and small parts can be tested. This method was applied widely: not only the conductive material defect detection, but also the measure of conductivity, permeability, heat treatment condition, and hardness and geometry of the material.

Eddy current flaw detector in actual use would affect by a number of factors, such as changes of ambient temperature, surface hardness, uneven mechanical rotating and jitter. The using of single eddy current probe is vulnerable to the above factors. In some serious cases it is unable to distinguish between defects and cracks. Therefore differential circuit must be used.

The distinguishing feature of eddy-current flaw detection is it functions with the conductive material but not necessarily the ferromagnetic material. But the effect with the ferromagnetic material is poor. Secondly, the work piece surface finish, smoothness and edge have great impact on eddy current testing. So eddy current testing often used for regular shape and smooth surface non-ferromagnetic work piece testing.

\section{b) Ultrasonic sensor}

Ultrasonic testing [4] is the most widely used method of non-destructive testing. It is applicable to a variety sizes of forging, rolling pieces, weld, and some of the casting. Iron, steel, non-ferrous metals and non-metallic, can be detected by ultrasound method, including the mechanical parts, structural parts, power plant equipment, hull, boilers, pressure and chemical containers. In physical properties, ultrasonic method can measure the thickness, material hardness, hardened layer depth, grain size, level and flow, residual stress and plastic bonding strength. But ultrasonic cannot test the specimen with rough surface and complex shape. And the method is often inaccurate on qualifying and quantifying the characterization of defect.

The high-frequency ultrasonic wave has short length. It rarely diffracts, and reflects on impurities or sub-interface. It has good directionality and small attenuation in the liquid and solid. These features make ultrasonic an important tool for nondestructive testing. Ultrasonic monitoring usually has two ways of method, penetration and reflection. The penetration method is shown in Figure 2. The reflection method is shown in Figure 3.

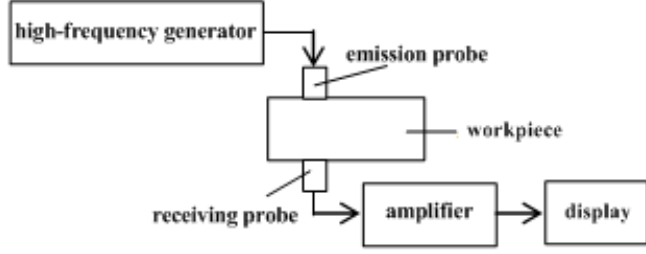

Figure 2. the penetration method

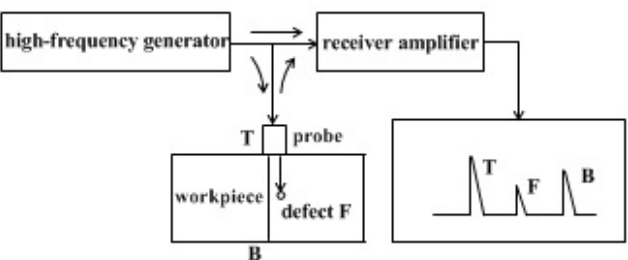

Figure 3. The reflection method

c) More comparisons

The eddy current NDT testing is a conventional method for detection of conductive material. Compared with ultrasonic detection, its measurement range is relatively small, and is susceptible to a variety of factors. And the eddy current excitation frequency directly affects the eddy current testing results. Generally, higher excitation frequency results in higher sensitivity. But higher excitation frequency causes the eddy current more concentrated on the surface of the specimen. The ultrasonic testing has the characteristics of wide adaptability, high sensitivity, less affected, not harmful to humans, flexible usage, lightweight equipment, low-cost, and immediate testing. It is suitable for working in a variety of environments and testing of running device and equipment. Although the ultrasonic testing is difficult to identify the type of defect, the ultrasonic spectrum analysis and ultrasonic holographic imaging method contribute to determine the defect. So compared to eddy current testing, ultrasonic testing is more applicable and active detection in the field of non-destructive flaw detection.

2) Monitoring of the insulator leakage

Insulator works long time in the environment, which contains strong electric field, high of sunshine, strong mechanical stress, high humidity and dirty matter (acid, ozone, dust, etc.).The insulator's insulation property will reduce quickly, when the salt density of the insulator's surface reaches to a certain level. This will damage the transmission line and even threat the whole distribution network. According to statistics, the proportion of uncertain line future on $110 \mathrm{kV}$ Power Line is up to $22 \%$, which is affecting the stable of the power system, and caused large economic losses. Over the years, there is lack of safe and stable observation system, which can check the insulators both in power line, and transformer stations without cut their power off. The existing insulator's insulation monitoring methods are as following: 


\section{a) Method of offline salt density monitoring:}

Take off the insulator from the using system, clean the surface with distilled water (or deionizer water), measure the conductivity and temperature of pre-obtained water, and convert the conductivity value to the standard temperature to $20{ }^{\circ} \mathrm{C}$, calculate the equivalents salt content and the equivalent salt with the relationship between conductivity and salt density.

b) Method of insulation resistance:

Connect the insulation resistance meter to the working insulator with a high resistance, set the meter to zero. Measured resistance value minus the added high resistance value is the resistance value of measuring insulator. This method is accuracy to detect the "zero value" or "low" insulator. However, for a large number of insulators detection workload is too difficult to carry out, and this methods must be used in good weather and the insulator surface condensation conditions, otherwise it's easy to miscarriage a good insulator to poor quality insulators.

\section{c) Method of distribution voltage determination:}

This method should compare the voltage distribution value [5] of the poor quality insulator with good insulator string. Detection Should also be carried out in the atmospheric environment, otherwise string voltage distribution on wet insulator will change, especially in the case of the surface of insulator is contamination and moisture, Voltage distribution on the insulator strings is not the capacitance distribution but the resistance distribution. Before the produce dry with previously, pollution and wet the more uniform insulator string voltage distribution is more uniform.

\section{d) Method of AC withstand:}

AC voltage test can test the effectiveness of the aforementioned methods and identify poor quality insulators, this method requires a high voltage test power and test product needs to be removed from the field. It is heavy workload.

\section{e) Method of ultrasonic testing:}

The method can measure the distribution of voltage when insulator strings is running. Because the ability of anti-jamming of the device is higher, and input capacitance is smaller (measured $\mathrm{l} \sim 2 \mathrm{pF}$ ), it can be measured in the $500 \mathrm{kV}$ transmission lines, and can ensure the accuracy of the measurements. This method still needs people to climb the tower and contact the probe with porcelain insulators. $500 \mathrm{kV}$ insulated operating rod is longer, that is very convenient on the detection of insulators - string.

f) Method of high voltage insulator surface leakage current and $\mathrm{PD}$ (Partial discharge) pulse:

To acquisition insulator surface leakage current and PD pulse [6] through phased uninterrupted. Setting the PD plus threshold, PD plus apparatus can recode each PD plus signal of various times which are added in the leak current.

What kind of insulator insulation monitoring methods will be chosen by the further experiment to explore.

3) Monitoring of environmental parameters and line
Environmental parameters include temperature, humidity, salinity, pressure, electromagnetic environment. This class of sensor is relatively mature.

\section{Data processing system}

The data content of the processing of the high-voltage tower monitoring system including the processing and storage of the various sensor measurements, the transmission desired signal to the signal transmission system by a specific protocol, the implementation of the communication system returns the command of the remote monitoring system.

The data processor includes dedicated chip of DSP, ARM, and other microprocessors, etc. To use high-performance signal processing DSP chips for processing at the research stage.

\section{Signal transmission system}

The existing signal transmission mode is satellite transmission, GPRS transmission and microwave transmission.

\section{E. Remote monitoring system}

The remote monitoring system is to complete the design of the monitoring software architecture and communication protocols.

\section{SUMMARY}

In the past 30 years, especially since the beginning of the 21st century, it is the reform of power system and national policies in Chinese that promote the rapid development of power grid. With the development of the smart grid, high-voltage tower monitoring system has shown its importance increasingly. The research about high-voltage tower monitoring system can not only improve the added value of the tower system and create core technology of independent intellectual property rights. It is also able to promote the development and application of smart grid technology.

\section{REFERENCES}

[1] Enose, Nampuraja, "A Unified management system for Smart Grid,”Source: 2011 IEEE PES International Conference on Innovative Smart Grid Technologies-India, pp.328-333, 2011.

[2] C. Cao, "The Application of ZigBee in The Monitoring System of Electric Current Arising Suddenly of High voltage Transmission Line,”CHINA SCI-TEC,2008, pp.848-851.

[3] Zhou, Fengxing, "Wireless remote monitoring system of high-voltage tower steel stress,"Source: Dianli Zidonghua Shebei/Electric Power Automation Equipment, v30, n11, pp. 115-117, November 2010.

[4] Mudge, P.J , “ Field application of the Teletest long-range ultrasonic testing technique," Non-Destructive Testing and Condition Monitoring, v 43, n 2, pp. 74-77, 2001.

[5] B. Zhang, "Voltage distribution along a long ceramic insulator string in a high-voltage tower window,"The International Journal for Computation and Mathematics in Electrical and Electronic Engineering, v29, n3, pp.811-823, January 1, 2010.

[6] Schwarz, R, "Partial discharge impulse behaviour in different insulating media,"Conference Record of IEEE International Symposium on Electrical Insulation, v 2007, p 306-309, 2007. 